\title{
SUMMATION OF SERIES VIA LAPLACE TRANSFORMS
}

\author{
A. SOFO
}

\begin{abstract}
We consider a forced differential difference equation and by the use of Laplace Transform Theory generate non-hypergeometric type series which we prove may be expressed in closed form.
\end{abstract}

\section{Introduction}

In a previous paper Sofo and Cerone [6] considered a differential-difference equation and by the use of Laplace Transform Theory were able to prove that the infinite sum

$$
S_{1}(R)=\sum_{n=0}^{\infty}\left(\begin{array}{c}
n+R-1 \\
n
\end{array}\right) \frac{b^{n} e^{-b(t-a n)}(t-a n)^{n+R-1}}{(n+R-1) !}
$$

may be represented in closed form.

For $R=1$, we have from (1.1), details of which may be seen in [2], that

$$
S_{1}(1)=\sum_{n=0}^{\infty} \frac{b^{n} e^{-b(t-a n)}(t-a n)^{n}}{n !}=\frac{1}{1+a b} .
$$

The result (1.2) was previously obtained, in different form, by Euler [3] in 1779 and later, in 1902, independently by Jensen [4].

However, the methods used by Euler and Jensen in obtaining (1.2) do not suggest a technique that would allow us to generalise (1.2) to obtain the result (1.1). The methods developed by Sofo and Cerone [5] in obtaining (1.2) did allow the author to generalise the result $S_{1}(1)$ to $S_{1}(R)$. We now apply similar methods as used in [5] or [2] to further generalise $S_{1}(R)$.

2000 Mathematics Subject Classification: Primary 05A15, Secondary 05A19. Servicio de Publicaciones. Universidad Complutense. Madrid, 2002 
The main purpose of this paper is therefore to prove that the sum

$$
S(k, b, a, t)=\sum_{n=0}^{\infty} \frac{b^{n k} e^{-b(t-a n)}(t-a n)^{n k+k-1}}{(n k+k-1) !}
$$

and its generalisation

$S(R, m, k, b, a, t)=\sum_{n=0}^{\infty}\left(\begin{array}{c}n+R-1 \\ n\end{array}\right) \frac{b^{n k} e^{-b(t-a n)}(t-a n)^{k(n+R)+m-1}}{(n k+R k+m-1) !}$

may be expressed in closed form.

\section{Technique}

Consider, for a well behaved function $f(t)$, the forced dynamical system with constant real coefficients $b$ and $c$, real delay parameter $a$, and all initial conditions at rest,

$$
\left\{\begin{array}{r}
c f(t-a)+\sum_{r=0}^{k}\left(\begin{array}{c}
k \\
r
\end{array}\right) b^{k-r} f^{(r)}(t)=w(t) ; \quad t>a \\
\sum_{r=0}^{k}\left(\begin{array}{c}
k \\
r
\end{array}\right) b^{k-r} f^{(r)}(t)=w(t) ; \quad 0<t \leq a .
\end{array}\right.
$$

In the system (2.1) $w(t)$ is a forcing term, $t$ is a real variable and $k$ is a positive integer. If we use the Laplace transform property

$$
\mathcal{L}(f(t-\alpha))=e^{-\alpha p} F(p)
$$

and the property of the Laplace transform of derivative functions, we obtain upon taking the Laplace transforms of (2.1)

$$
F(p)=\frac{W(p)}{(p+b)^{k}+c e^{-a p}},
$$

where $F(p)$ and $W(p)$ are the Laplace transforms of $f(t)$ and $w(t)$ respectively.

If we rewrite $(2.2)$ as

$$
F(p)=\frac{W(p)}{(p+b)^{k}}\left[\frac{1}{1+\frac{c e^{-a p}}{(p+b)^{k}}}\right]
$$


and expand the square bracket as a series, we have

$$
F(p)=\sum_{n=0}^{\infty} \frac{W(p)\left(-c e^{-a p}\right)^{n}}{(p+b)^{n k+k}} .
$$

To bring out the essential features of our results we may choose the forcing term $w(t)=\delta(t)$, the Dirac delta function, such that $W(p)=1$. Substituting for $W(p)$ into (2.3) and taking the inverse Laplace transform, we have

$$
f(t)=\sum_{n=0}^{\infty} \frac{(-c)^{n} e^{-b(t-a n)}(t-a n)^{n k+k-1}}{(n k+k-1) !} H(t-a n),
$$

where $H(x)$ is the unit Heaviside step function. The inverse of (2.2), a solution of the system (2.1) by Laplace transform theory, may also be written as

$$
f(t)=\frac{1}{2 \pi i} \int_{\gamma-i \infty}^{\gamma+i \infty} e^{p t} F(p) d p
$$

for an appropriate choice of $\gamma$ such that all the zeros of the characteristic function

$$
g_{1}(p)=(p+b)^{k}+c e^{-a p}
$$

are contained to the left of the line in the Bromwich contour, and $F(p)$ is defined by (2.2). Now, by the residue theorem

$$
f(t)=\sum \text { residues of }\left(e^{p t} F(p)\right),
$$

which suggests the solution of $f(t)$ may be written in the form

$$
f(t)=\sum_{\rho} Q_{\rho} e^{p_{\rho} t}
$$

where the sum is over all the characteristic zeros $p_{\rho}$ of $g_{1}(p)$ and $Q_{\rho}$ is the contribution of the residues in $F(p)$ at $p=p_{\rho}$. The zeros of the characteristic function (2.5), under certain restrictions are all distinct. The poles of the expression (2.2) depend on the zeros of the characteristic function (2.5), namely, the zeros of $g_{1}(p)$. The $k$ dominant distinct roots 
$\xi_{\nu}, \nu=0,1,2, \ldots, k-1$, of $g_{1}\left(\xi_{\nu}\right)=0$ are defined as the ones with the greatest real part and therefore we have that asymptotically

$$
f(t) \backsim \sum_{\nu=0}^{k-1} Q\left(\xi_{\nu}\right) e^{\xi_{\nu} t}
$$

From (2.4) and (2.6)

$$
f(t)=\sum_{n=0}^{[t / a]} \frac{(-c)^{n} e^{-b(t-a n)}(t-a n)^{n k+k-1}}{(n k+k-1) !} \backsim \sum_{\nu=0}^{k-1} Q\left(\xi_{\nu}\right) e^{\xi_{\nu} t} .
$$

where $[x]$ represents the integer part of $x$ and the residue contribution $Q\left(\xi_{\nu}\right)$, is given by

$$
Q\left(\xi_{\nu}\right)=\lim _{p \rightarrow \xi_{\nu}}\left[\left(p-\xi_{\nu}\right) F(p)\right], \quad \nu=0,1,2, \ldots, k-1
$$

and $\xi_{\nu}$ are defined as the $k$-dominant distinct zeros of the characteristic function (2.5). To simplify the algebra let us take $c+b^{k}=0$, which allows one dominant zero of the characteristic function

$$
g_{k}(p)=(p+b)^{k}-b^{k} e^{-a p}
$$

with $k+a b>0$, to occur at the origin. The condition $k+a b \neq 0$ will ensure the distinct nature of the zeros of (2.9).

From the above considerations and the suggestion of (2.7), we may state our main theorem as follows.

\section{The Main Theorem}

Theorem 1. Let

$$
\begin{gathered}
T_{n}(k, b, a, t)=\frac{b^{n k} e^{-b(t-a n)}(t-a n)^{n k+k-1}}{(n k+k-1) !} \text { and } \\
S(k, b, a, t)=\sum_{n=0}^{\infty} T_{n}(k, b, a, t)
\end{gathered}
$$


which is convergent for all values of $k, b, a$ and $t$ in the region

$$
\left|(a b)^{k} e^{a b}\right|<\left(k e^{-1}\right)^{k}
$$

Then

$$
S(k, b, a, t)=\sum_{\nu=0}^{k-1} Q\left(\xi_{\nu}\right) e^{\xi_{\nu} t},
$$

where $Q\left(\xi_{\nu}\right)$ is defined in (2.8) and $\xi_{\nu}$ are the $k$ dominant distinct zeros of the characteristic function (2.9), and $F(p)$ in (2.8) is given by (2.2) with $W(p)=1$ and $c=-b^{k}$. The inequality (3.3) is obtained by using the ratio test on (3.1).

The following two lemmas, regarding the location of dominant zeros, will be useful in the proof of Theorem 1 .

Lemma 1. The characteristic function (2.9) has $k$ simple dominant zeros lying in the region $\Gamma:|p|<\frac{k+a b}{a} ; a, b>0$ and $k$ is a positive integer.

Proof. We have previously defined a dominant zero as the one with the greatest real part. It is known, see [1], that (2.9) has an infinite number of zeros lying in the left (or right) half plane. By using some straightforward algebra, it can be shown that (2.9) has at most three (and at least one) real zeros with restriction (3.3) one of which is at the origin, $\xi_{0}=0$. Applying Rouche's theorem [8] it is required to show that $|A(w)|>|B(w)-A(w)|$ for $w=p+b, A(w)=w^{k}, B(w)=$ $w^{k}-b^{k} e^{a b-a w}$ in the region $\Gamma^{\prime}:|w|<\frac{k+2 a b}{a}$. Now $A(w)$ has $k$ zeros lying in the region $\Gamma^{\prime}$ and since $\left|w^{k}\right|>\left|-b^{k} e^{a b-a w}\right|$ implies that $(k+2 a b)^{k}>$ $(a b)^{k}$; then $B(w)$ has $k$ dominant zeros lying in the region $\Gamma^{\prime}$ and hence (2.9) has $k$ dominant zeros lying in $\Gamma$.

Lemma 2. The characteristic function

$$
q_{j}(p)=p+b-b e^{(2 \pi i j-a p) / k}
$$

for $j=0,1,2,3, \ldots, k-1$ has one dominant zero for each $j$ lying in the region $\Gamma$ as defined in Lemma 1.

Proof. Now, $A_{1}(w)=w$ has one dominant zero lying in the region $\Gamma^{\prime}$ and $B_{1}(w)=w-b e^{(2 \pi i j+a b-a w) / k}$. Therefore $|w|>\left|-b e^{(2 \pi i j+a b-a w) / k}\right|$ 
implies that $(k+2 a b)>a b$, hence $B_{1}(w)$ has one zero lying inside the region $\Gamma^{\prime}$ and it follows that for $j=0,1,2,3, \ldots, k-1,(3.5)$ has one dominant zero lying in the region $\Gamma$.

Proof. (Theorem 1). Firstly, we evaluate $Q\left(\xi_{\nu}\right)$ from (2.8) and from (3.4) we may write

$$
\begin{aligned}
S(k, b, a, t) & =\sum_{n=0}^{\infty} \frac{b^{n k} e^{-b(t-a n)}(t-a n)^{n k+k-1}}{(n k+k-1) !} \\
& =\sum_{\nu=0}^{k-1} \frac{e^{\xi_{\nu} t}}{\left(b+\xi_{\nu}\right)^{k-1}\left(k+a b+a \xi_{\nu}\right)} .
\end{aligned}
$$

The characteristic function (2.9) may be expressed as the product of factors such that,

$$
g_{k}(p)=(p+b)^{k}-b^{k} e^{-a p}=\prod_{j=0}^{k-1}\left(p+b-b e^{(2 \pi i j-a p) / k}\right)=\prod_{j=0}^{k-1} q_{j}(p) .
$$

Lemmas 1 and 2 show that the dominant zeros, $\alpha_{j}$, of $q_{j}\left(\alpha_{j}\right)$ for each $j=0,1,2,3, \ldots, k-1$ are the same as the $k$ dominant zeros of $g_{k}(p)$. Using (2.8), the contribution $\Omega\left(\alpha_{j}\right)$ to each of the factors $q_{j}\left(\alpha_{j}\right)$ is

$$
\begin{aligned}
\Omega\left(\alpha_{j}\right) & =\lim _{p \rightarrow \alpha_{j}}\left[\left(p-\alpha_{j}\right) F_{j}(p)\right] \\
& =\lim _{p \rightarrow \alpha_{j}}\left[\frac{p-\alpha_{j}}{q_{j}(p)}\right]=\frac{k}{k+a b+a \alpha_{j}}
\end{aligned}
$$

and using this result, we have from (1.2) that

$$
\sum_{n=0}^{\infty} \frac{\left(b e^{2 \pi i j / k}\right)^{n} e^{-b\left(t-\frac{a n}{k}\right)}\left(t-\frac{a n}{k}\right)^{n}}{n !}=\frac{k e^{\alpha_{j} t}}{k+a b+a \alpha_{j}}
$$

for each $k=1,2,3, \ldots$ and $j=0,1,2,3, \ldots, k-1$. Note that the sum (3.7) may in fact be a complex number. The summation of all the $k$ dominant 
zeros, for each of the factors $q_{j}\left(\alpha_{j}\right)$ implies from (3.7) that

$$
\begin{aligned}
\sum_{j=0}^{k-1} \frac{k e^{\alpha_{j} t}}{k+a b+a \alpha_{j}} & =\sum_{j=0}^{k-1} \sum_{n=0}^{\infty} \frac{\left(b e^{2 \pi i j / k}\right)^{n} e^{-b\left(t-\frac{a n}{k}\right)}\left(t-\frac{a n}{k}\right)^{n}}{n !} \\
& =\sum_{n=0}^{\infty} \frac{b^{n} e^{-b\left(t-\frac{a n}{k}\right)}\left(t-\frac{a n}{k}\right)^{n}}{n !} \sum_{j=0}^{k-1} e^{2 \pi i j n / k}
\end{aligned}
$$

Rescaling the infinite sum, by putting $n=\left(n^{*}+1\right) k$, (and then renaming $n^{*}$ as $n$ ) gives the result,

$$
k b^{k} \sum_{n=-1}^{\infty} \frac{b^{n k} e^{-b(t-a n-a)}(t-a n-a)^{n k+k}}{(n k+k) !}=\sum_{j=0}^{k-1} \frac{k e^{\alpha_{j} t}}{k+a b+a \alpha_{j}} .
$$

Now letting $y=t-a$ and, from $q_{j}\left(\alpha_{j}\right)$, using the fact that $e^{a \alpha_{j}}=$ $\left(\frac{b}{b+\alpha_{j}}\right)^{k}$ then

$$
\sum_{n=0}^{\infty} \frac{b^{n k} e^{-b(y-a n)}(y-a n)^{n k+k}}{(n k+k) !}=\sum_{j=0}^{k-1} \frac{e^{\alpha_{j} y} e^{-b(a+y)}}{\left(b+\alpha_{j}\right)^{k}\left(k+a b+a \alpha_{j}\right) b^{k}}
$$

Differentiating (3.8) with respect to $y$, which is permissible within the radius of convergence (3.3), gives after some algebraic manipulation

$$
\sum_{n=0}^{\infty} \frac{b^{n k} e^{-b(y-a n)}(y-a n)^{n k+k-1}}{(n k+k-1) !}=\sum_{j=0}^{k-1} \frac{e^{\alpha_{j} y}}{\left(b+\alpha_{j}\right)^{k-1}\left(k+a b+a \alpha_{j}\right)} .
$$

Renaming $y$ as $t$ shows that (3.9) is the same as (3.6) since by Lemmas 1 and $2, \alpha_{j}=\xi_{\nu}$ for $j=0,1,2,3, \ldots, k-1 ; \nu=0,1,2,3, \ldots, k-1$, and therefore Theorem 1 is proved.

Some examples are now given to illustrate the above theorem.

\subsection{Examples}

$(i)$. For $k$ even there are 2 real dominant distinct zeros and $(k-2)$ complex conjugate zeros of the characteristic function (2.9) that need to 
be considered for determining the right hand side of (3.6). Consider, in particular, the case $k=2$, then

$$
\sum_{n=0}^{\infty} \frac{b^{2 n} e^{-b(t-a n)}(t-a n)^{2 n+1}}{(2 n+1) !}=\sum_{\nu=0}^{1} \frac{e^{\xi_{\nu} t}}{\left(b+\xi_{\nu}\right)\left(2+a b+a \xi_{\nu}\right)} .
$$

For $(a, b, t)=(.1,2,2)$ then $\left(\xi_{0}, \xi_{1}\right)=(0,-4.5053)$ and the sum takes the value, to four significant digits, .2272.

(ii). For $k$ odd there are 3 real dominant distinct zeros and $(k-1)$ complex conjugate zeros of the characteristic function (2.9) that need to be considered for determining the right hand side of (3.6). Consider, in particular, $k=3$, in this case there will be one real zero $\xi_{0}$ and two complex conjugate zeros $\xi_{1}=(x+i y), \bar{\xi}_{1}=(x-i y)$ and $\xi_{\nu}$ satisfies $\left(\xi_{\nu}+b\right)^{3}-b^{3} e^{-a \xi_{\nu}}=0, \nu=0,1,2$. Hence we have

$$
\begin{aligned}
& \sum_{n=0}^{\infty} \frac{b^{3 n} e^{-b(t-a n)}(t-a n)^{3 n+2}}{(3 n+2) !} \\
= & \frac{e^{\xi_{0} t}}{\left(b+\xi_{0}\right)^{2}\left(3+a b+a \xi_{0}\right)} \\
+ & \frac{2 e^{x t}\left[\left(x_{2} x_{4}-x_{3} x_{5}\right) \cos y t+\left(x_{2} x_{5}+x_{3} x_{4}\right) \sin y t\right]}{\left(x_{1}^{2}+y^{2}\right)^{2}\left(x_{5}^{2}+x_{4}^{2}\right)}
\end{aligned}
$$

where $x_{1}=(x+b), x_{2}=x_{1}^{2}-y^{2}, x_{3}=2 y x_{1}, x_{4}=3+a x_{1}$ and $x_{5}=a y$. For $(a, b, t)=(.8,1,2)$ then

$$
\left(\xi_{0}, \xi_{1}, \bar{\xi}_{1}\right)=(0,-1.2193+1.3668 i,-1.2193-1.3668 i)
$$

and the sum takes the value, to four significant digits, .2769. It is also of some interest to note that for $j=1, q_{1}(p)$ of (3.5) takes the dominant zero value, $\xi_{1}=-1.2193+1.3668 i$ and hence the sum (3.7) takes the value $-0.0616+0.0606 i$ to four significant figures.

Again the previous results (3.6) may be extended in various directions, we briefly mention one extension.

\section{Extension}

Consider, for a well behaved function $f(t)$, the forced dynamical system with constant real coefficient $b$, real delay parameter $a$, and all initial 
conditions at rest,

$$
\left.\begin{array}{r}
\sum_{j=0}^{R}\left(\begin{array}{c}
R \\
R-j
\end{array}\right) b^{k(R-j)} \sum_{r=0}^{j k}\left(\begin{array}{c}
j k \\
r
\end{array}\right) b^{j k-r} f^{(r)}(t-(R-j) a) \\
=w(t) ; \quad t>R a \\
\sum_{r=0}^{R k}\left(\begin{array}{c}
R k \\
r
\end{array}\right) b^{R k-r} f^{(r)}(t)=w(t) ; \quad 0<t \leq R a
\end{array}\right)
$$

In the system (4.1) $w(t)$ is a forcing term, $t$ a real variable, and $R$ and $k$ are positive integers. Let $w(t)=\frac{e^{-b t} t^{m-1}}{(m-1) !}$, for $m=1,2,3, \ldots$ and taking the Laplace transform of (4.1) we have

$$
F(p)=\frac{1}{(p+b)^{m}\left((p+b)^{k}-b^{k} e^{-a p}\right)^{R}}
$$

By the methods of the previous Section 3 we finally obtain

$$
\begin{aligned}
& \sum_{n=0}^{\infty}\left(\begin{array}{c}
n+R-1 \\
n
\end{array}\right) \frac{b^{n k} e^{-b(t-a n)}(t-a n)^{n k+R k+m-1}}{(n k+R k+m-1) !} \\
= & \sum_{r=0}^{m-1} \frac{e^{-b t} t^{m-r-1} P_{m, r}(-b)}{(m-r-1) !}+\sum_{\nu=0}^{k-1} \sum_{\mu=0}^{R-1} \frac{e^{-\xi_{\nu} t} t^{R-\mu-1} Q_{R, \mu}\left(\xi_{\nu}\right)}{(R-\mu-1) !}
\end{aligned}
$$

where

$$
\begin{gathered}
r ! P_{m, r}(-b)=\lim _{p \rightarrow-b}\left[\frac{d^{r}}{d p^{r}}\left((p+b)^{m} F(p)\right)\right] ; r=0,1,2, \ldots,(m-1), \\
\mu ! Q_{R, \mu}\left(\xi_{\nu}\right)=\lim _{p \rightarrow \xi_{\nu}}\left[\frac{d^{\mu}}{d p^{\mu}}\left(\left(p-\xi_{\nu}\right)^{R} F(p)\right)\right] ; \mu=0,1,2, \ldots,(R-1),
\end{gathered}
$$

$F(p)$ is defined by $(4.2)$ and $\xi_{\nu}, \nu=0,1,2,3, \ldots, k-1$ are the $k$ dominant zeros of the characteristic function $(2.9)$. For $(R, k, m)=(2,2,3)$ we 
have, from ( 4.3)

$$
\begin{aligned}
& \sum_{n=0}^{\infty}(n+1) \frac{b^{2 n} e^{-b(t-a n)}(t-a n)^{2 n+6}}{(2 n+6) !} \\
= & \frac{1}{2\left(b^{2} e^{a b}\right)^{3}}\left[b^{2} e^{a b} t(t+4 a)+4\left(1+a^{2} b^{2} e^{a b}\right)\right] \\
& +\sum_{\nu=0}^{1} \frac{e^{\xi_{\nu} t}}{\left(b+\xi_{\nu}\right)^{2}\left(2\left(b+\xi_{\nu}\right)+a b^{2} e^{\left.-a \xi_{\nu}\right)^{2}}\right.} \\
& {\left[t-\frac{2}{b+\xi_{\nu}}-\frac{2-a^{2} b^{2} e^{-a \xi_{\nu}}}{2\left(b+\xi_{\nu}\right)+a b^{2} e^{-a \xi_{\nu}}}\right], }
\end{aligned}
$$

where $\xi_{\nu}$ are the two dominant zeros of $\left(\xi_{\nu}+b\right)^{2}-b^{2} e^{-a \xi_{\nu}}=0$. The degenerate case of $a=0$, implies that the transcendental function (2.9) reduces to a polynomial in $p$ of degree $k$. Specifically for $(a, R, k, m)$ $=(0,2,2,3)$ we have the identity

$$
\sum_{n=0}^{\infty}(n+1) \frac{(b t)^{2 n+6}}{(2 n+6) !}=\frac{1}{2}\left[(b t)^{2}+4+b t \sinh (b t)-4 \cosh (b t)\right] .
$$

\section{Concluding Remarks}

We have proved, by function theoretic methods, that the two nonhypergeometric sums, $S(k, b, a, t)$ and $S(R, m, k, b, a, t)$ may be represented in closed form.

By a similar approach as that described in Section 2, Sofo and Cerone [5] and Sofo [7] were able to represent infinite Fibonacci type sums in closed form.

\section{References}

[1] R. Bellman and K. L. Cooke, Differential Difference Equations. Academic Press, New York, 1963.

[2] P. Cerone and A. Sofo, Summing Series Arising From Integro-DifferentialDifference Equations, Australian Math. Soc. Series B, Vol. 41, pp. 473-486, 2000. 
[3] L. Euler, De serie Lambertina plurimisque eius insignibus proprietatibus. Acta Acad. Sci. Petropolitanae, pp.29-51, 1779; II(1783).

[4] J.L.W.V. Jensen, Sur une identite d'Abel et sur d'autres formules analogues. Acta Mathematica,Vol. 26, pp.307-313, 1902.

[5] A. Sofo and P. Cerone, Generalisation of Euler's Identity, Bull. Australian Math. Soc., Vol. 58, pp. 359-371, 1998.

[6] A. Sofo and P. Cerone, On a Fibonacci Related Series, The Fibonacci Quarterly, Vol. 36, pp. 211-215, 1998.

[7] A. Sofo, An Extension of Jensen's Form, Bull. Australian Math. Soc., Vol. 63, pp.279-298, 2001.

[8] H. Tagaki, Queueing Analysis, A Foundation of Performance Evaluation, Vol.1, Vacation and Priority System, Part 1. North Holland, Amsterdam, 1991.

School of Communiations and Informatics

Victoria University of Technology

PO Box 14428

Melbourne City MC

Victoria 8001, Australia.

E-mail: sofo@matilda.vu.edu.au

http://sci.vu.edu.au/scistaff.html

Recibido: 2 de Abril de 2001

Revisado: 30 de Noviembre de 2001 